\title{
Previous maternal infection protects offspring from enterovirus infection and prevents experimental diabetes development in mice
}

\author{
P. G. Larsson • T. Lakshmikanth • E. Svedin • C. King • \\ M. Flodström-Tullberg
}

Received: 17 August 2012 / Accepted: 2 January 2013 /Published online: 24 January 2013

(C) Springer-Verlag Berlin Heidelberg 2013

\begin{abstract}
Aims/hypothesis Enterovirus (e.g. Coxsackie B virus serotypes [CVBs]) infections may be associated with development of type 1 diabetes. Studies conducted in several European countries have, however, shown an inverse correlation between the incidence of type 1 diabetes and the prevalence of enterovirus infections. These findings could in part be explained by an extension of the poliovirus hypothesis, suggesting that the absence of maternally transferred antibodies protecting offspring from early infection increases the risk for diabetes development. Experimental evidence supporting this hypothesis in type 1 diabetes is, however, lacking. As maternally transferred protection from infection is a crucial component of the extended poliovirus hypothesis, we here tested the hypothesis that previously infected females transfer protection against infection and diabetes to offspring.
\end{abstract}

Electronic supplementary material The online version of this article (doi:10.1007/s00125-013-2834-z) contains peer-reviewed but unedited supplementary material, which is available to authorised users.

P. G. Larsson • T. Lakshmikanth • E. Svedin •

M. Flodström-Tullberg $(\bowtie)$

The Center for Infectious Medicine, Department of Medicine HS,

Karolinska Institutet, Karolinska University Hospital Huddinge

F59, SE-141 86,

Stockholm, Sweden

e-mail: Malin.Flodstrom-Tullberg@ki.se

T. Lakshmikanth

The Department of Microbiology, Tumor and Cell Biology,

Karolinska Institutet, Stockholm, Sweden

C. King

Department of Immunology, The Garvan Institute for Medical Research, Faculty of Medicine, The University of New South Wales, Sydney, Australia
Methods The induction of CVB-specific maternal antibodies and transfer of protection from virus infection, replication and development of virus-induced diabetes to offspring was assessed using NOD and Socs 1 -transgenic NOD mice. Results Infected mice produced neutralising antibodies to CVB. Offspring from infected females were positive for neutralising antibodies and were strongly protected from both infection and experimental diabetes.

Conclusions/interpretation Our study shows that maternally transferred antibodies protect offspring from enterovirus infection and virus-induced diabetes. This suggests that the absence of maternally provided protection increases the risk for severe outcomes after an enterovirus infection in offspring. Moreover, our findings may have implications for the design of prospective studies aimed at investigating the possible role of enterovirus infections in the aetiology of human type 1 diabetes.

Keywords Coxsackievirus $\cdot$ Enterovirus $\cdot$ Maternal antibodies $\cdot$ NOD mouse $\cdot$ Poliovirus hypothesis $\cdot$ Socs 1 -tg mouse - Type 1 diabetes
Abbreviations
CVB Coxsackievirus, serotype B
KRV Kilham rat virus
PFU Plaque-forming unit
p.i. Post infection
tg Transgenic

\section{Introduction}

Susceptibility to type 1 diabetes is regulated by both genetic and environmental factors. Epidemiological studies have 
suggested that infections with enteroviruses, in particular those of the coxsackie B virus (CVB) serotypes, may be linked to islet autoimmunity and development of type 1 diabetes [1-5]. Other observations suggesting that infections with these viruses have a causative role in diabetes development come from studies that found enteroviruses more frequently in pancreases [6-8] and, in some studies, also gut biopsies [9-11], from patients with type 1 diabetes compared with healthy controls. Some enteroviruses, including the CVBs, are pancreatropic and can infect human beta cells in vitro, often with a detrimental outcome (e.g. [6, 12]). Furthermore, experimental animal models have indicated that enterovirus infections can cause direct beta cell damage $[13,14]$ and alter disease progression in the NOD mouse model [15-17].

The incidence of type 1 diabetes varies considerably between different countries [18]. Based upon the observations linking enterovirus infections to the development of type 1 diabetes [1-5], it would be expected that such infections are more common in countries with a high disease incidence compared with those with an intermediate or low incidence. In contrast to this assumption, epidemiological studies conducted in different geographical regions of Europe have demonstrated that the frequency of enterovirus infections in the background population shows an inverse correlation to the incidence of type 1 diabetes [18-20].

The observation that the prevalence of enterovirus infections is low in countries with a high incidence of type 1 diabetes may support an argument against a causal role for these viruses in human type 1 diabetes. However, a parallel to this inverse correlation has been described for poliovirus, a virus also belonging to the enterovirus genus [21]. Before the beginning of the 20th century, poliovirus infections were endemic and the risk of paralytic complications (poliomyelitis) was low. Since the end of the 19th century, improved hygienic standards and public sanitation has led to a decrease in the frequency of poliovirus infections in the developed world. Paradoxically, this led to a dramatic increase of individuals developing paralytic poliomyelitis. A likely explanation to this development is that herd immunity declined in parallel with the reduced circulation of poliovirus [21]. Indeed, in the past, when poliovirus infections were common, children encountered the virus early in life under the protection of maternal antibodies, leading to mild infections and immunity. When poliovirus infections became less frequent, the proportion of women with immunity decreased and few children that encountered the virus early in life were protected by maternally transferred antibodies. Thus, the lack of maternally transferred protection or a later exposure to poliovirus allowed the virus to spread more vigorously, resulting in increased tissue damage and paralytic poliomyelitis in some susceptible individuals [21].
The so-called poliovirus hypothesis has thus provided the basis for a related hypothetical model explaining the increased incidence of type 1 diabetes in countries with a low frequency of enterovirus infections. This model, proposed by Viskari et al, states that a low frequency of enterovirus infections in the background population increases the risk for severe complications such as beta cell damage and type 1 diabetes in infected young individuals [22]. The heightened risk for diabetes development may be explained by lower levels and a narrower serotype-spectrum of maternally transferred antibodies, or by children being exposed to enteroviruses later in childhood when the protective effect of maternal antibodies has waned [5, 22-25]. Indirect evidence for this hypothesis has recently been published, demonstrating that high levels of maternal enterovirus antibodies in serum and breast milk correlate with a reduced frequency of such infections in children [26]. Moreover, a study by Tirabassi et al showed that rats born from mothers previously infected with Kilham rat virus (KRV) were protected from KRV-induced diabetes [27]. This study did not, however, investigate the role of maternal antibodies in this protection, and direct evidence for maternally mediated protection against enterovirus infection and diabetes is lacking.

In this study, we set out to determine whether maternal antibodies protect offspring from virus infection and genetically susceptible mice from virus-induced diabetes. Using the NOD and Socs 1 -transgenic (tg) mouse models, we assessed maternal protection against enterovirus infection and enterovirusinduced diabetes in genetically susceptible offspring.

\section{Methods}

Viruses Mycoplasma-free stocks of CVB3 Nancy (CVB3) and B4 Edward 2 (CVB4) (originally kindly provided by G. Frisk, Uppsala University, Sweden) were generated in HeLa cells and partially sequenced after amplification. All sample and virus dilutions were done in RPMI 1640 or PBS.

Virus titration and neutralisation assays Virus titrations were performed using HeLa cells and a standard plaque assay, with lower detection limits of 100 plaque-forming units $(\mathrm{PFU}) / \mathrm{ml}$, and $50 \mathrm{PFU} / \mathrm{g}$ in blood and pancreatic tissue, respectively. Virus neutralisation assays were conducted using a plaque-reduction assay where diluted serum ( $1: 8$ to $1: 2,048$ or $1: 10$ to $1: 1,000$ ) was mixed and incubated with $30 \mathrm{PFU}$ of $\mathrm{CVB} 3$ for $1 \mathrm{~h}$ at $37^{\circ} \mathrm{C}$ followed by overnight incubation at room temperature. Pooled serum from uninfected NOD mice was used as a negative control and internal standard to which all included samples were normalised. All neutralisation data are presented as compared with the infectivity of negative control serum, described in figures as 'infection (\% of controls)'. Human antibody-positive serum 
was used as positive control serum. All samples were analysed in duplicate.

Animal husbandry NOD mice and Socs1-tg mice were bred and housed in a specific-pathogen-free environment at Karolinska Institutet, Stockholm, Sweden. The generation and screening of the Socs 1 - $\operatorname{tg}$ mouse model on a NOD background have been described previously [13, 14, 28, 29]. In brief, these mice express Socs1 under the control of the human insulin promotor and, as a result of a failing interferon-induced antiviral defence in the beta cells, develop diabetes within 511 days after infection with either CVB3 or CVB4 $[13,14]$. Socs 1 -tg offspring were generated through heterozygous breeding with Socs 1 -tg males and NOD females. All animal experiments were approved by the local ethics committee and conducted in accordance to the NIH principles of laboratory animal care and the Swedish law.

In vivo infections, breeding of previously infected females and infections of offspring Due to the low infection reproducibility using the oral route, all in vivo infections were induced intraperitoneally using $10^{2}-10^{3}$ PFU CVB3 Nancy diluted in $100-200 \mu \mathrm{l}$ sterile PBS. Mice treated with PBS alone served as controls. Blood (diluted 1:1 in $12 \mathrm{mmol} / \mathrm{l}$ EDTA in PBS) was routinely saved on days 0 and 3 post infection (p.i.) for measurements of viraemia.

To investigate whether maternal antibodies are transferred to offspring and whether offspring are protected against infection and diabetes development, female NOD mice were infected with CVB3 $\left(1 \times 10^{2}-5 \times 10^{2} \mathrm{PFU}\right)$ at an age of 4 6 weeks. Serum was harvested on days 0 and 14 p.i. for determination of the presence of neutralising antibodies against CVB3. Breeding of confirmed antibody-positive and uninfected (antibody-negative) females with Socs 1 -tg males was set up $2-3$ weeks p.i. All progeny ( $3.9 \pm 0.6$ weeks old) from naive and previously infected females were genotyped and infected intraperitoneally with $10^{3}$ PFU CVB3, a dose of virus known to induce diabetes in Socs1-tg mice (P.G. Larsson and M. Flodström-Tullberg, unpublished observations). Serum samples were saved before infection and blood samples were saved on day 3 p.i. for measurement of neutralising antibodies and viraemia, respectively. Some non-tg NOD mice (one or two pups from a representative number of mothers) were killed on day 3 p.i. for determination of pancreatic viral titres.

Diabetes monitoring and sample collection Development of diabetes was monitored up to day 28 p.i. by tail-vein blood glucose readings under non-fasting conditions. Mice were considered diabetic when presenting with either one blood glucose reading $>21 \mathrm{mmol} / \mathrm{l}$ or two consecutive readings $>13 \mathrm{mmol} / \mathrm{l}$. Mice that developed diabetes were killed on the day of diagnosis while the other mice were kept until day 28 p.i. Pancreases were saved for histological analysis (fixed in
$4 \%$ formaldehyde in PBS for $24 \mathrm{~h})$ and plaque assays $\left(-80^{\circ} \mathrm{C}\right.$ until further processing).

Adoptive serum transfer To obtain serum containing neutralising antibodies, NOD mice were mock infected or infected one to three times with $10^{2}-10^{3} \mathrm{PFU}$ CVB3 at 6 9 weeks of age, to achieve high serum titres of neutralising antibodies. Serum was harvested by heart puncture 14 days post last infection. Female and male Socs 1 -tg mice $(8$ 12 weeks old) received pooled and heat-inactivated $\left(56^{\circ} \mathrm{C}\right.$ for $30 \mathrm{~min}$ ) sera from infected or mock-infected mice (400 $\mu \mathrm{l} /$ mouse injected intraperitoneally) $24 \mathrm{~h}$ before infection with CVB3 $\left(10^{3} \mathrm{PFU}\right)$. Blood and serum samples were harvested before injection of inactivated sera, and a serum sample was saved before infection with CVB3. Blood was saved on day 3 p.i. for viraemia measurements. Because Socs 1 -tg mice develop diabetes between day 5 and 11 p.i. $[13,14]$, blood glucose levels were measured up to day 12 p.i. Diabetic or moribund mice were killed. Pancreases were saved for histological analysis (fixed in $4 \%$ formaldehyde in PBS for $24 \mathrm{~h}$ ). Development of diabetes was monitored according to the criteria described above, with the exception of two mice that became moribund without accompanying hyperglycaemia. In these two mice the loss of beta cells and islet integrity was assessed by immunohistochemistry (insulin and glucagon staining of pancreatic sections).

Histology and immunohistochemistry Tissue sections were prepared and stained as previously described [30]. Analyses of stained tissue sections were performed blinded by two independent observers, as previously described [13].

Statistical analysis All statistical analyses were performed using the Graphpad Prism 5 software (GraphPad Software, La Jolla, CA, USA). A $p$ value $<0.05$ was considered statistically significant.

\section{Results}

CVB3 infection induces neutralising antibodies We first infected male and female NOD mice with CVB3 to determine whether NOD mice raise neutralising antibodies to CVBs. A blood sample was drawn on day 3 p.i. and all infected mice had viraemia (see Electronic supplementary material [ESM] Fig. 1a); no virus was detectable in the blood from PBS-treated control mice. Serum was harvested from the productively infected and mock-infected mice on day 14 p.i. All infected mice had high titres of neutralising antibodies, whereas serum from PBS-treated controls showed no neutralising activity (ESM Fig. 1b). Further, the antibody-positive sera failed to neutralise CVB4, showing sero-specificity for CVB3 (data not shown). 

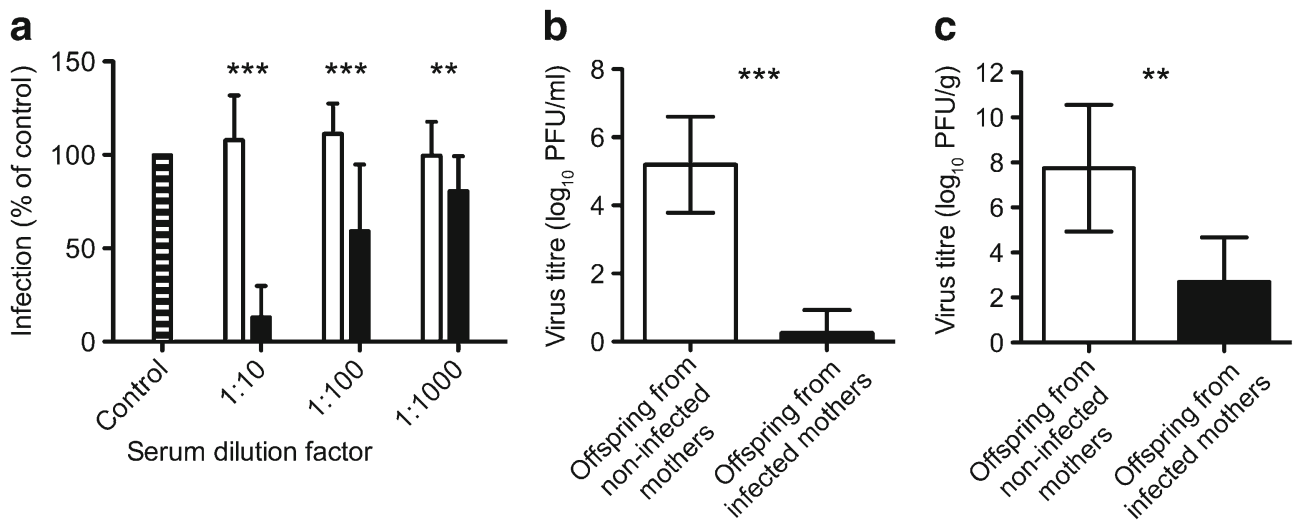

Fig. 1 Offspring from female NOD mice previously infected with CVB3 have neutralising antibodies and are protected from infection. (a) Titres of CVB3-neutralising antibodies measured in serum harvested from male and female offspring from four uninfected female NOD mice (white bars, $n$ of offspring $=35$ ) and three infected female NOD mice (black bars, $n=27$ ) at weaning, compared with negative control serum (striped bar). The ability of each serum sample to neutralise a known amount of virus was measured by a standard reduction plaque assay, and the neutralising effect was compared with

Offspring from previously infected females have CVB3neutralising antibodies Next, we set out to determine whether neutralising maternal antibodies are transferred to offspring. Naive or previously infected antibody-positive NOD females were bred with naive males. Serum was saved from all progeny at weaning (age $27 \pm 4$ days). Sera from offspring originating from infected females contained neutralising antibodies $(n=27)$, as indicated by the ability to neutralise CVB3 (Fig. 1a). No neutralising activity was observed in serum from offspring originating from naive females ( $n=35$, Fig. 1a)

Offspring from previously infected females are protected from viraemia and have lower levels of replicating virus in the pancreas To investigate whether previous infection of mothers provides offspring with protection from virus infection, we infected offspring from both naive $(n=11)$ and infected $(n=$ 23) NOD females with CVB3 after weaning (age $27 \pm 4$ days). If protected, we expected to see a significant reduction of viraemia, pancreatic virus titres and pancreatic damage in offspring originating from infected females compared with offspring from naive females. Blood and pancreases harvested on day 3 p.i. showed that offspring from infected females had significantly lower virus titres in blood (Fig. $1 \mathrm{~b} ; p<0.001$ ) and pancreases (Fig. 1c; $p<0.01$ ) compared with offspring from naive females. Histological analyses of formalin-fixed pancreases confirmed that offspring from infected females had less damage to the exocrine tissue than pancreases saved from offspring of naive females (data not shown).

Previous maternal infection protects genetically susceptible offspring from virus-induced diabetes Our results clearly and presented as percentage of infection obtained using the same amount of virus and an internal negative serum control. (b) Virus titres in blood 3 days p.i. in offspring from two naive female NOD mice (white bar, $n=11$ ) and from three infected female mice (black bar, $n=$ 23). (c) Virus titres in pancreas 3 days p.i. in offspring from two naive NOD female mice (white bar, $n=6$ ) and from three infected females (black bar, $n=10$ ). Data are presented as means \pm SD. ${ }^{* *} p<0.01$ and $* * * p<0.001$, two-way ANOVA with Bonferroni correction (a) and Mann-Whitney $U$ tests $(\mathbf{b}, \mathbf{c})$

indicated that maternal antibodies protect offspring from virus infection. Thus, we wished to use an experimental model to determine whether maternal antibodies also protect offspring from enterovirus-induced diabetes (Fig. 2a). Based upon numerous findings in humans suggesting that beta cells are directly infected by enteroviruses [6-8] and that this, at least in part, explains the role for a viral infection in the disease process, we selected a model for enterovirusinduced diabetes that is dependent on the access of enterovirus to the pancreatic beta cell, the Socs 1 -tg mouse model $[13,14]$. We bred previously infected antibody-positive NOD females and uninfected control NOD females with naive Socs 1 -tg male mice. Socs 1 -tg offspring were infected with CVB3. While Socs 1-tg offspring from naive females developed diabetes rapidly after infection, Socs 1 -tg offspring from infected females were strongly protected from virus-induced diabetes (Fig. $2 \mathrm{~b} ; p<0.001$ ). An immunohistochemical analysis of pancreases revealed significant islet and exocrine tissue destruction in mice originating from naive females (Fig. 2c). However, no destruction of either islets or exocrine tissue was observed in pancreases from offspring originating from infected females (Fig. 2d).

Adoptive serum transfer protects mice from CVB3-induced diabetes Although we awaited viral clearance in the CVB3challenged females before breeding with Socs 1 -tg males it cannot be completely excluded that the maternal infection could have affected the offsprings' susceptibility to infection by other means than the passive transfer of neutralising antibodies. To confirm the protection of passively transferred antibodies we next performed adoptive serum transfers. To this end, male and female Socs 1 -tg mice verified to 


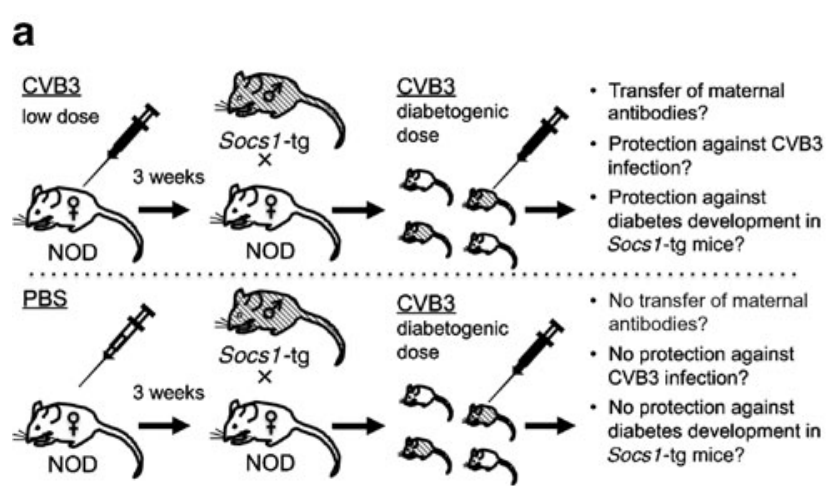

Fig. 2 Socs 1 -tg offspring from female mice previously infected with CVB3 are protected from CVB3-induced diabetes and pancreatic damage. (a) Model illustrating the experimental set-up. (b) Diabetes incidence in male and female Socs 1 -tg offspring from four naive female mice (black line, $n=19$ ) and three infected female mice (dashed line, $n=10)$ after infection with CVB3. (c, d). Immunohistochemical staining

be negative for CVB3-neutralising antibodies (data not shown), received heat-inactivated serum pooled from either uninfected (antibody-negative) or CVB3-infected (antibody-positive) mice. Twenty-four hours later serum was drawn for measurement of neutralising antibodies and then the mice were infected with CVB3. Mice receiving serum from previously infected mice were positive for neutralising antibodies in serum, while mice receiving serum from mock-infected mice were negative (Fig. 3a). Moreover, mice that received antibody-positive sera had significantly lower levels of replicating virus in their circulation day 3 p.i. compared with mice that received antibody-negative sera (Fig. 3b). Infected mice receiving antibody-positive sera were also protected from developing virus-induced diabetes after CVB3 infection (Fig. 3c).

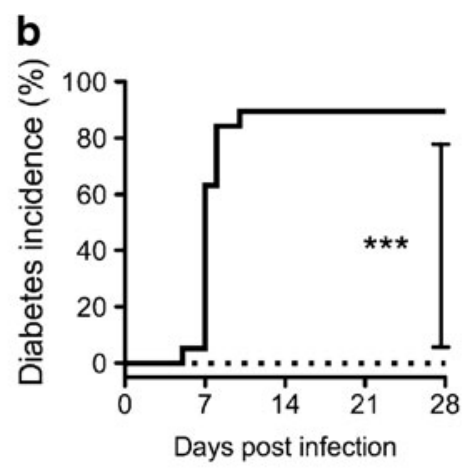

C

d
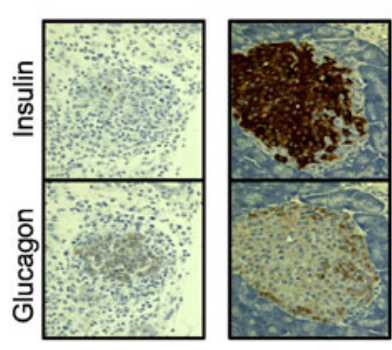

for insulin and glucagon in the pancreas from a CVB3-infected Socs 1-tg mouse originating from a naive mother and that developed diabetes day 7 p.i. (c), and a non-diabetic infected Socs 1 -tg mouse originating from an infected mother, day 28 p.i. (d). Histological images are representative of each condition. ${ }^{* * *} p<0.001$, logrank (Mantel-Cox) test (b)

A histological analysis revealed severe pancreatic destruction in all mice receiving antibody-negative serum $(n=4$, data not shown), while the pancreases of mice receiving antibodypositive serum were completely intact or showed only minimal tissue damage ( $n=5$, data not shown). Collectively, these results clearly demonstrate that passive immunisation by antibodies protects against enterovirus infection and, in Socs 1 -tg mice, virus-induced diabetes.

\section{Discussion}

In this study we demonstrate that NOD mice raise high titres of sero-specific neutralising antibodies in response to an enterovirus (CVB3) infection and that offspring from previously
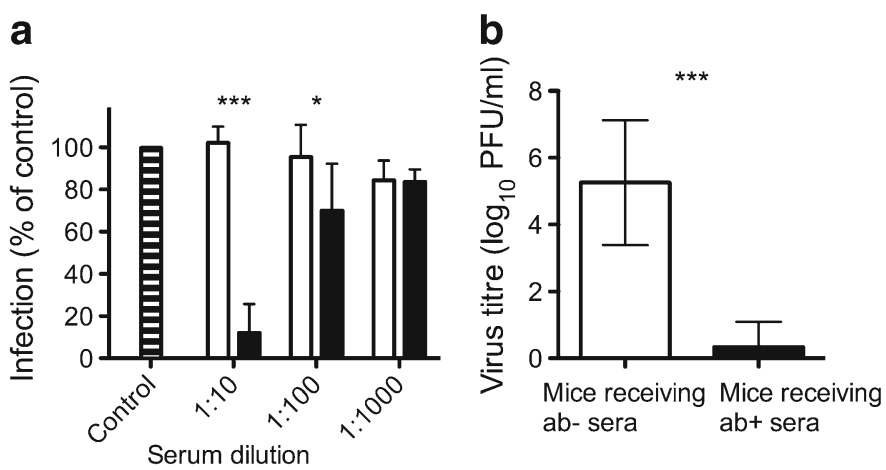

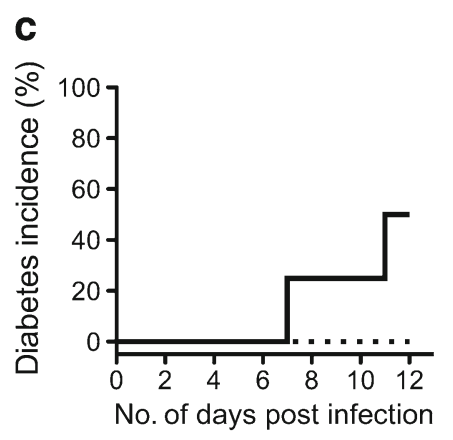

Fig. 3 Adoptive transfer of antibody-positive sera protects Socs 1 -tg offspring from virus infection and diabetes development. (a) Antibody levels in serum of male and female Socs 1 -tg mice offspring $24 \mathrm{~h}$ after adoptive serum transfers with antibody-negative sera (white bars, $n=4$ ) or antibody-positive sera (black bars, $n=5$,) compared with negative control (striped bar). The ability of each serum sample to neutralise a known amount of virus was measured by a standard reduction plaque assay, and the neutralising effect was compared with and presented as percentage of infection obtained using the same amount of virus and an internal negative serum control. (b) Viraemia in offspring that received antibody-negative sera $\left(\mathrm{ab}^{-}, n=4\right)$ or antibody-positive sera $(\mathrm{ab}+, n=$ 5), day 3 p.i. with CVB3. (c) Diabetes incidence in Socs 1 -tg mice offspring that received antibody-negative sera (black line, $n=4$ ) or antibody-positive sera (dotted line, $n=5$ ) $24 \mathrm{~h}$ before CVB3 infection. Data are presented as means \pm SD. ${ }^{*} p<0.05$ and ${ }^{* * *} p<0.001$, two-way ANOVA with Bonferonni correction (a), Mann-Whitney $U$ test (b) and logrank (Mantel-Cox) test (c) 
infected females acquire antibodies with neutralising capacity. We also show that offspring from females that have been infected with virus before pregnancy are protected against enterovirus infection and, in the case of Socs 1 -tg mice, virus-induced diabetes development. Collectively, our results support an important role for maternally transferred antibodies in this protection and strengthens the hypothesis that a mother's history of enterovirus infections before pregnancy may regulate susceptibility to severe outcomes of infant enterovirus infections. As discussed more in detail below, our studies may also have important implications for the development of vaccination strategies aimed at investigating the role of enteroviruses in the aetiology of human type 1 diabetes.

In 2000, Viskari et al postulated an extension to the socalled poliovirus hypothesis, to explain the inverse relationship between the prevalence of enterovirus infections and incidence of type 1 diabetes [22]. Indirect evidence for the extended poliovirus hypothesis has been published. First, as an indication of a declining 'herd immunity' to enteroviruses, maternal antibody levels have decreased in many European countries over the past 20 years [20]. Second, the frequency of maternal antibodies to different enteroviruses is higher in countries with a low or intermediate incidence of type 1 diabetes than in countries with a high incidence of the disease [20], suggesting a relationship between low herd immunity to enteroviruses and high risk for type 1 diabetes development. Finally, high levels of maternal enterovirus antibodies in serum and breast milk correlate with a reduced frequency of such infections in children [26]. Although our study does not provide proofof-concept for the extended poliovirus hypothesis, it provides direct evidence that a previous enterovirus infection in mothers affords protection from infection and lowers the risk for virus-induced diabetes in Socs 1 -tg offspring.

Only a few animal models for enterovirus-induced autoimmune type 1 diabetes have been described. Diabetes development after enterovirus infection in the most commonly used experimental model, the NOD mouse, is dependent on the already established prediabetic phase including insulitis [15-17, 31], which in the NOD mouse occurs after maternally transferred antibodies may have waned [32]. This is in contrast with humans in whom islet autoantibodies may already appear before the disappearance of maternal antibodies [23, 33, 34]. Thus, the NOD mouse model may not be the first choice for studies investigating the role of maternal antibodies in protection from infection and diabetes development. In the absence of a suitable model for enterovirus-triggered autoimmune type 1 diabetes in young mice the present study made use of the Socs 1 -tg mouse model $[13,14]$. Using this model we could clearly show that previous maternal infection led to protection from direct virus-induced beta cell damage. Virus-induced beta cell damage is an event that may be of importance for the autoimmune process to be initiated or accelerated in humans [6-8], although unlikely to be as dramatic as in the Socs 1 -tg model $[13,14]$. However, since diabetes development in the Socs 1 -tg mouse model is not dependent on autoreactive T cells [13], future studies should investigate whether maternal protection against virus-induced $\mathrm{T}$ cell mediated autoimmune diabetes can be studied in alternative experimental systems, such as the LCMV/RIP-GP/NP mouse models [35, 36] or the KRV/LEW.1WR1 rat model [27].

Collectively, the extended poliovirus hypothesis and the results presented here may be viewed as an extension of the hygiene hypothesis, which states that hosts who are less exposed to infectious agents are at higher risk for developing autoimmune disorders and allergies [37, 38]. Indeed, it may not be the lack of enterovirus infections in the primary host that is detrimental, but rather the lack of maternal infectious exposure that is a contributing factor for disease development in offspring. The more enterovirus serotypes a prospective mother has been exposed to before pregnancy, the better the child is protected against such serotypes [26] and their possible diabetogenic effect. This extends the hygiene hypothesis to include also a lack of microbial exposure to prospective mothers. The data we present here highlights an important role for maternal antibodies in protecting offspring from potentially diabetogenic enterovirus infections during early life, and implies that the increasingly cleaner environment in the developed world puts children at higher risk for developing type 1 diabetes.

As a final note, it is important to point out that our study neither proves nor disproves the hypothesis that enteroviruses play a role in type 1 diabetes. Evidence in support of a causative role for a pathogen such as an enterovirus in type 1 diabetes would be the demonstration that a vaccine prevents the disease in humans. The development of an enterovirus vaccine aimed at providing protection from infection is, however, a challenging task due to the existence of numerous serotypes in the enterovirus genus and the risk for undesired cross-reactive immune responses to self-antigens.

Another important issue is insufficient vaccine effectiveness in neonates and young infants due to their partially immature immune system [39]. This bottleneck cannot be ignored given the fact that enterovirus infections are common already before the age of 3 months [40]. The findings we present here offer a potential solution to the problem of poor vaccine efficacy in newborns by suggesting that maternal immunity is passively transferred to the offspring, providing immediate protection from infections. Hence, an enterovirus vaccine could potentially be given to women before pregnancy occurs or, if the vaccine is considered safe for the growing fetus, during pregnancy, something that is already done in some countries (e.g. with vaccines against influenza, tetanus, diphtheria and pertussis). The presence of neutralising antibodies in the offspring already from birth 
would assure protection from infection until the child can be successfully vaccinated.

In summary, we have shown that passively transferred immunity protects offspring from virus-induced damage, including beta cell damage, and diabetes in genetically predisposed mice. Our data provide experimental proof of an important role for maternal antibodies in protecting offspring from infection and severe outcomes of enterovirus infection. This knowledge could be implemented in future prospective enterovirus vaccine studies aimed at preventing infections as early as from birth and thereby investigating the role of enterovirus infections in development of human type 1 diabetes.

Acknowledgements We would like to express our gratitude to $\mathrm{H}$. Hyöty and collaborators S. Tauriainen and S. Oikarinen (Department of Virology, University of Tampere, Finland) for their help in setting up the neutralisation assay and screening of our virus stocks. K. Lind (Karolinska Institutet) is greatly acknowledged for discussions regarding this project.

Funding This study was supported by a European Foundation for the Study of Diabetes/Novo Nordisk grant, Karolinska Institutet, the Strategic Research Programme in Diabetes at Karolinska Institutet, the Swedish Child Diabetes Foundation, the Swedish Diabetes Association Research Foundation and the Swedish Research Council. M. Flodström-Tullberg is in part supported by a VINNMER fellowship from VINNOVA, Sweden.

Contribution statement PGL designed the experimental approach, acquired and analysed all data and wrote the manuscript. TL and ES acquired data, contributed to discussion and edited the manuscript. CK designed the experimental approach and edited the manuscript. MF-T designed the experimental approach, analysed the data and wrote the manuscript. All authors gave their approval of the final version of the manuscript.

Duality of interest The authors declare that there is no duality of interest associated with this manuscript.

\section{References}

1. Richer MJ, Horwitz MS (2009) Coxsackievirus infection as an environmental factor in the etiology of type 1 diabetes. Autoimmun Rev 8:611-615

2. Hober D, Sauter P (2010) Pathogenesis of type 1 diabetes mellitus: interplay between enterovirus and host. Nat Rev Endocrinol 6:279-289

3. Stene LC, Oikarinen S, Hyoty H et al (2010) Enterovirus infection and progression from islet autoimmunity to type 1 diabetes: the Diabetes and Autoimmunity Study in the Young (DAISY). Diabetes 59:3174-3180

4. Yeung WC, Rawlinson WD, Craig ME (2011) Enterovirus infection and type 1 diabetes mellitus: systematic review and metaanalysis of observational molecular studies. BMJ 342:d35

5. Ghazarian L, Diana J, Simoni Y, Beaudoin L, Lehuen A (2013) Prevention or acceleration of type 1 diabetes by viruses. Cell Mol Life Sci 70:239-255
6. Ylipaasto P, Klingel K, Lindberg AM et al (2004) Enterovirus infection in human pancreatic islet cells, islet tropism in vivo and receptor involvement in cultured islet beta cells. Diabetologia 47:225-239

7. Dotta F, Censini S, van Halteren AG et al (2007) Coxsackie B4 virus infection of beta cells and natural killer cell insulitis in recent-onset type 1 diabetic patients. Proc Natl Acad Sci U S A 104:5115-5120

8. Richardson SJ, Willcox A, Bone AJ, Foulis AK, Morgan NG (2009) The prevalence of enteroviral capsid protein vp1 immunostaining in pancreatic islets in human type 1 diabetes. Diabetologia 52:1143-1151

9. Oikarinen M, Tauriainen S, Honkanen T et al (2008) Detection of enteroviruses in the intestine of type 1 diabetic patients. Clin Exp Immunol 151:71-75

10. Oikarinen M, Tauriainen S, Oikarinen S et al (2012) Type 1 diabetes is associated with enterovirus infection in gut mucosa. Diabetes 61:687-691

11. Mercalli A, Lampasona V, Klingel K et al (2012) No evidence of enteroviruses in the intestine of patients with type 1 diabetes. Diabetologia 55:2479-2488

12. Hultcrantz M, Huhn MH, Wolf $M$ et al (2007) Interferons induce an antiviral state in human pancreatic islet cells. Virology 367:92101

13. Flodstrom M, Maday A, Balakrishna D, Cleary MM, Yoshimura A, Sarvetnick N (2002) Target cell defense prevents the development of diabetes after viral infection. Nat Immunol 3:373-382

14. Flodstrom M, Tsai D, Fine C, Maday A, Sarvetnick N (2003) Diabetogenic potential of human pathogens uncovered in experimentally permissive beta-cells. Diabetes 52:2025-2034

15. Serreze DV, Ottendorfer EW, Ellis TM, Gauntt CJ, Atkinson MA (2000) Acceleration of type 1 diabetes by a coxsackievirus infection requires a preexisting critical mass of autoreactive $\mathrm{T}$ cells in pancreatic islets. Diabetes 49:708-711

16. Tracy S, Drescher KM, Chapman NM et al (2002) Toward testing the hypothesis that group B coxsackieviruses (CVB) trigger insulin-dependent diabetes: inoculating nonobese diabetic mice with CVB markedly lowers diabetes incidence. J Virol 76:12097-12111

17. Serreze DV, Wasserfall C, Ottendorfer EW et al (2005) Diabetes acceleration or prevention by a coxsackievirus B4 infection: critical requirements for both interleukin-4 and gamma interferon. $\mathrm{J}$ Virol 79:1045-1052

18. The DIAMOND Project Group (2006) Incidence and trends of childhood type 1 diabetes worldwide 1990-1999. Diabet Med 23:857-866

19. Viskari H, Ludvigsson J, Uibo R et al (2004) Relationship between the incidence of type 1 diabetes and enterovirus infections in different European populations: results from the EPIVIR project. J Med Virol 72:610-617

20. Viskari H, Ludvigsson J, Uibo R et al (2005) Relationship between the incidence of type 1 diabetes and maternal enterovirus antibodies: time trends and geographical variation. Diabetologia 48:1280-1287

21. Nathanson N, Martin JR (1979) The epidemiology of poliomyelitis: enigmas surrounding its appearance, epidemicity, and disappearance. Am J Epidemiol 110:672-692

22. Viskari HR, Koskela P, Lonnrot M et al (2000) Can enterovirus infections explain the increasing incidence of type 1 diabetes? Diabetes Care 23:414-416

23. Zinkernagel RM (2001) Maternal antibodies, childhood infections, and autoimmune diseases. N Engl J Med 345:13311335

24. Zinkernagel RM (2003) On natural and artificial vaccinations. Annu Rev Immunol 21:515-546 
25. Gamble DR (1980) The epidemiology of insulin dependent diabetes with particular reference to the relationship of virus infection to its etiology. Epidemiol Rev 2:49-70

26. Sadeharju K, Knip M, Virtanen SM et al (2007) Maternal antibodies in breast milk protect the child from enterovirus infections. Pediatrics 119:941-946

27. Tirabassi RS, Guberski DL, Blankenhorn EP et al (2010) Infection with viruses from several families triggers autoimmune diabetes in LEW*1WR1 rats: prevention of diabetes by maternal immunization. Diabetes 59:110-118

28. Flodstrom-Tullberg M, Yadav D, Hagerkvist R et al (2003) Target cell expression of suppressor of cytokine signaling-1 prevents diabetes in the NOD mouse. Diabetes 52:2696-2700

29. Hultcrantz M, Jacobson S, Hill NJ, Santamaria P, FlodstromTullberg M (2009) The target cell response to cytokines governs the autoreactive T cell repertoire in the pancreas of NOD mice. Diabetologia 52:299-305

30. Flodstrom-Tullberg M, Hultcrantz M, Stotland A et al (2005) RNase L and double-stranded RNA-dependent protein kinase exert complementary roles in islet cell defense during coxsackievirus infection. J Immunol 174:1171-1177

31. Horwitz MS, Bradley LM, Harbertson J, Krahl T, Lee J, Sarvetnick N (1998) Diabetes induced by Coxsackie virus: initiation by bystander damage and not molecular mimicry. Nat Med 4:781-785

32. Appleby P, Catty D (1983) Transmission of immunoglobulin to foetal and neonatal mice. J Reprod Immunol 5:203-213
33. Ziegler AG, Hummel M, Schenker M, Bonifacio E (1999) Autoantibody appearance and risk for development of childhood diabetes in offspring of parents with type 1 diabetes: the 2-year analysis of the German BABYDIAB Study. Diabetes 48:460-468

34. Kimpimaki T, Kupila A, Hamalainen AM et al (2001) The first signs of beta-cell autoimmunity appear in infancy in genetically susceptible children from the general population: the Finnish Type 1 Diabetes Prediction and Prevention Study. J Clin Endocrinol Metab 86:4782-4788

35. Ohashi PS, Oehen S, Buerki K et al (1991) Ablation of "tolerance" and induction of diabetes by virus infection in viral antigen transgenic mice. Cell 65:305-317

36. Oldstone MB, Nerenberg M, Southern P, Price J, Lewicki H (1991) Virus infection triggers insulin-dependent diabetes mellitus in a transgenic model: role of anti-self (virus) immune response. Cell 65:319-331

37. Strachan DP (1989) Hay fever, hygiene, and household size. BMJ 299:1259-1260

38. Bach JF (2001) Protective role of infections and vaccinations on autoimmune diseases. J Autoimmun 16:347-353

39. PrabhuDas M, Adkins B, Gans H et al (2011) Challenges in infant immunity: implications for responses to infection and vaccines. Nat Immunol 12:189-194

40. Salur L, Oikarinen S, Tauriainen S, Mandel M, Hyoty H, Uibo R (2011) Enterovirus infections in young infants: are children still protected by maternal antibodies? Hum Vaccines 7:966-971 